\title{
Search for the QCD Critical Point with Fluctuations of Conserved Quantities in Relativistic Heavy-Ion Collisions at RHIC
}

\author{
Xiaofeng Luo ${ }^{1, a}$ \\ ${ }^{1}$ Institute of Particle Physics and Key Laboratory of Quarks \& Lepton Physics (MoE), Central China Normal \\ University, Wuhan, China, 430079
}

\begin{abstract}
Fluctuations of conserved quantities are predicted to be sensitive observables to probe the QCD phase transition and critical point in heavy-ion collisions. Experimentally, various cumulants (up to fourth order) of net-proton (proxy for net-baryon (B)), net-kaon (proxy for net-strangeness (S)), and net-charge (Q) multiplicity distributions of $\mathrm{Au}+\mathrm{Au}$ collisions at $\sqrt{s_{N N}}=7.7 \sim 200 \mathrm{GeV}$ has been measured by the STAR and PHENIX experiments from the first phase of beam energy scan program at the Relativistic Heavy Ion Collider. In this paper, i will discuss those experimental results and the corresponding physics implications.
\end{abstract}

\section{Introduction}

The Quantum Chromodynamics (QCD) phase structure can be displayed in the two dimensional phase diagram (temperature, $T$ vs. baryon chemical potential, $\mu_{B}$ ). Finite temperature Lattice QCD calculations has shown that at zero $\mu_{B}\left(\mu_{B}=0\right)$ region, it is a crossover transition between hadronic phase and quark-gluon phase. At large $\mu_{B}$ region, the QCD based models predicted that the QCD phase transition is of the first order and there should exist a so called QCD Critical Point (CP) as the endpoint of the first order phase boundary [1]. Due to sign problem at finite $\mu_{B}$ region, it is difficult to precisely determine the location of the $\mathrm{CP}$ or even its existence $[2,3]$. One of the main goals of the Beam Energy Scan (BES) program at the Relativistic Heavy Ion Collider (RHIC) is to search for the CP in heavy-ion collisions experiment. Experimental confirmation of the existence of the CP will be an excellent verification of QCD theory in the non-perturbative region and a milestone of exploring the QCD phase structure. To vary the temperature $(T)$ and baryon chemical potential $\left(\mu_{B}\right)$ of the nuclear matter created in heavy-ion collisions [4], physicist has tuned the Au+Au nuclear colliding energies from high to low values, this allows us to access a broad region of the QCD phase diagram.

Theretically, fluctuations of conserved quantities, such as net-baryon (B), net-charge (Q) and netstrangeness (S), have been long time predicted to be sensitive to the correlation length [5-7] and directly connected to the susceptibilities computed in the first principle Lattice QCD calculations [8, 9]. Thus, it can serve as a powerful observables to study the phase transition and search for the CP in heavy-ion collisions. During the first phase of the RHIC BES (2010 to 2014), the STAR experiment has measured the cumulants (up to the fourth order) of net-proton (proton minus anti-proton number, proxy of net-baryon [10]), net-charge and net-kaon multiplicity distributions in Au+Au collisions at

\footnotetext{
ae-mail: xfluo@mail.ccnu.edu.cn
} 
$\sqrt{s_{\mathrm{NN}}}=7.7,11.5,14.5,19.6,27,39,62.4$ and $200 \mathrm{GeV}$. In those energies, the data of $14.5 \mathrm{GeV}$ is taken in the year 2014, 19.6, $27 \mathrm{GeV}$ are taken in the year 2011, and the other energies are collected in the years 2010. In this paper, we will discuss the experimental results from the STAR experiment for the fluctuations of conserved quantities. The corresponding physics implications will be discussed.

The paper is organized as follows: In the second section, we will give a brief discussion of the techniques used in the moment analysis. The experimental results and discussion will be presented in the third section. Finally, we will give a summary and outlook.

\section{Experimental Observables}

\subsection{Definition}

Particle multiplicity fluctuations can be characterized by the cumulants of the multiplicity distributions. It can be calculated as

$$
C_{1}=\langle N\rangle, C_{2}=\left\langle(\delta N)^{2}\right\rangle, C_{3}=\left\langle(\delta N)^{3}\right\rangle, C_{4}=\left\langle(\delta N)^{4}\right\rangle-3\left\langle(\delta N)^{2}\right\rangle^{2}
$$

Where $N$ is particle or net-particle number and the $\langle N\rangle$ is average over events, $\delta N=N-\langle N\rangle$. With the definition of cumulants, we can also define mean $(M)$, variance $\left(\sigma^{2}\right)$, skewness $(S)$ and kurtosis $(\kappa)$ as:

$$
M=C_{1}, \sigma^{2}=C_{2}, S=\frac{C_{3}}{\left(C_{2}\right)^{\frac{3}{2}}}, \kappa=\frac{C_{4}}{\left(C_{2}\right)^{2}}
$$

In addition, the moments product $\kappa \sigma^{2}$ and $S \sigma$ can be expressed in terms of the ratios of cumulants:

$$
\kappa \sigma^{2}=\frac{C_{4}}{C_{2}}, S \sigma=\frac{C_{3}}{C_{2}}, \sigma^{2} / M=\frac{C_{2}}{C_{1}}
$$

The ratios of cumulants are independent on system volume. The statistical errors of those cumulants and cumulants ratios are estimated by the Delta theorem [11, 12]. In general, the statistical errors strongly depend on the shape of the distributions, especially the width. For gaussian distributions, the statistical errors of cumulants $\left(C_{n}\right)$ can be approximated as $\operatorname{error}\left(C_{n}\right) \propto \sigma^{n} /\left(\sqrt{N} \epsilon^{n}\right)$, where $\sigma$ is the measured width of the distribution, $N$ represents the number of events and $\epsilon$ is the particle detection efficiency.

\subsection{Data Analysis Techniques}

Experimentally, one cannot directly measure the collision centrality and/or initial collision geometry of the system of two nuclei. The collision centrality is usually determined by comparing the measured particle multiplicity with the Glauber Monte Carlo simulations. It is denoted as a percentage value (for e.g. $0-5 \%, 30-40 \%, \ldots 70-80 \%$ ) for a collection of events to represent the fraction of the total cross section. This in general can cause two undesirable effects in the multiplicity fluctuations within finite centrality bin. One is the so called centrality bin width effect, which is caused by volume variation within a finite centrality bin size and the other one is centrality resolution effect depending on the number of particles used in the centrality definitions. Detector effects such as finite detection efficiency will dilute the origin signals and proper methods are needed to be applied to recover the signals. Finally, statistical errors estimation for the high order fluctuations is not straightforward and very complicated. Although it is difficult measurement, a set of analysis techniques were built up to measure the various order fluctuations of conserved quantities in heavy-ion collisions. Those 
include: (1) Novel centrality determination to account for the effects of centrality resolution and auto-correlation [13]. (2) Centrality bin width correction [13, 14]. (3) Efficiency correction and error estimation $[11,12,15,16]$. Those techniques are very important to address the background effects and extract the real physics fluctuation signals from the observables. The centrality bin width correction is to address the volume fluctuations effects on the higher order moments of conserved quantities distributions within a finite width centrality bin. It is also called centrality bin width effect. This effect needs to be eliminated, as an artificial centrality dependence could be introduced by it. To do this, the centrality bin width correction is applied to calculate the various moments of particle multiplicities distributions in one wide centrality bin. Experimentally, the smallest centrality bin is determined by a single value of particle multiplicity. Experimental measurements are usually analysed for a wider centrality bin (a range of particle multiplicity), such as $0-5 \%, 30-40 \%, \ldots .70-80 \%$,etc., to reduce statistical errors. To eliminate the centrality bin width effects, we calculate the various order cumulants $\left(C_{n}\right)$ for each single particle multiplicity within one wider centrality bin and weighted averaged by the corresponding number of events in that multiplicity.

$$
C_{n}=\sum_{r=N_{1}}^{N_{2}} \omega_{r} C_{n}^{r}
$$

where the $n_{r}$ is the number of events for multiplicity value $r$ and the corresponding weight for the multiplicity $r, \omega_{r}=n_{r} / \sum_{r=N_{1}}^{N_{2}} n_{r} . N_{1}$ and $N_{2}$ are the lowest and highest multiplicity values for one centrality bin. For more details, one can see [13].

\section{Experimental Results}

The STAR experiment published the energy dependence of cumulants (up to fourth order) of netproton [17] and net-charge [18] multiplicity distributions in $\mathrm{Au}+\mathrm{Au}$ collisions at $\sqrt{s_{\mathrm{NN}}}=7.7,11.5$, 19.6, 27, 39, 62.4 and $200 \mathrm{GeV}$. In the CPOD2014 and QM2015 conference [19-21], the STAR experiment reported the new net-proton fluctuation results of wider transverse momentum coverage $\left(0.4<p_{T}<2 \mathrm{GeV} / \mathrm{c}\right)$ by identifying proton and anti-proton with time of flight detector. Meanwhile, the preliminary results of cumulants of net-kaon distributions in Au+Au collision at $\sqrt{s_{\mathrm{NN}}}=7.7,11.5$, 14.5, 19.6, 27, 39, 62.4 and $200 \mathrm{GeV}$ are shown in the QM 2015 conference [21].

Figure 1 panel (a), (c), (d) show the energy dependence of $\kappa \sigma^{2}$ of net-charge, net-kaon and netproton multiplicity distributions in $\mathrm{Au}+\mathrm{Au}$ collisions measured by the STAR experiment for two centralities $(0-5 \%$ and $70 \%-80 \%)$ at $\sqrt{s_{\mathrm{NN}}}=7.7,11.5,14.519 .6,27,39,62.4$ and $200 \mathrm{GeV}$. Fluctuations of net-charge in $\mathrm{Au}+\mathrm{Au}$ collisions measured by the PHENIX experiment are shown in the panel (b) of Fig. 1. It shows that the net-charge and net-kaon fluctuations measured by the STAR experiment are with larger statistical errors than the errors of net-proton fluctuations. This is because the statistical errors of various order cumulants depend on the width $(\sigma)$ of the multiplicity distributions $\left(\left(\operatorname{error}\left(C_{n}\right) \propto \sigma^{n} /\left(\sqrt{N} \epsilon^{n}\right)\right)\right)$ and the net-charge distributions are much wider than net-proton and net-kaon. On the other hand, due to decay of kaons, the efficiency of kaon $(\sim 40 \%)$ is much lower than proton $(\sim 80 \%)$, this also leads to larger statistical errors for net-kaon fluctuations. The Skellam (Poisson) expectations shown in the figure as dashed lines with unity value reflect a system of totally uncorrelated, statistically random particle production. It predicts the $\kappa \sigma^{2}$ to be unity for Skellam expectations as well as in the hadron resonance gas model. For the net-proton results, a clear nonmonotonic energy dependence is observed for $\kappa \sigma^{2}$ in the most central ( 0-5\%) Au+Au collisions with a minimum around 19.6 and $27 \mathrm{GeV}$. This non-monotonic behavior cannot be described by various 

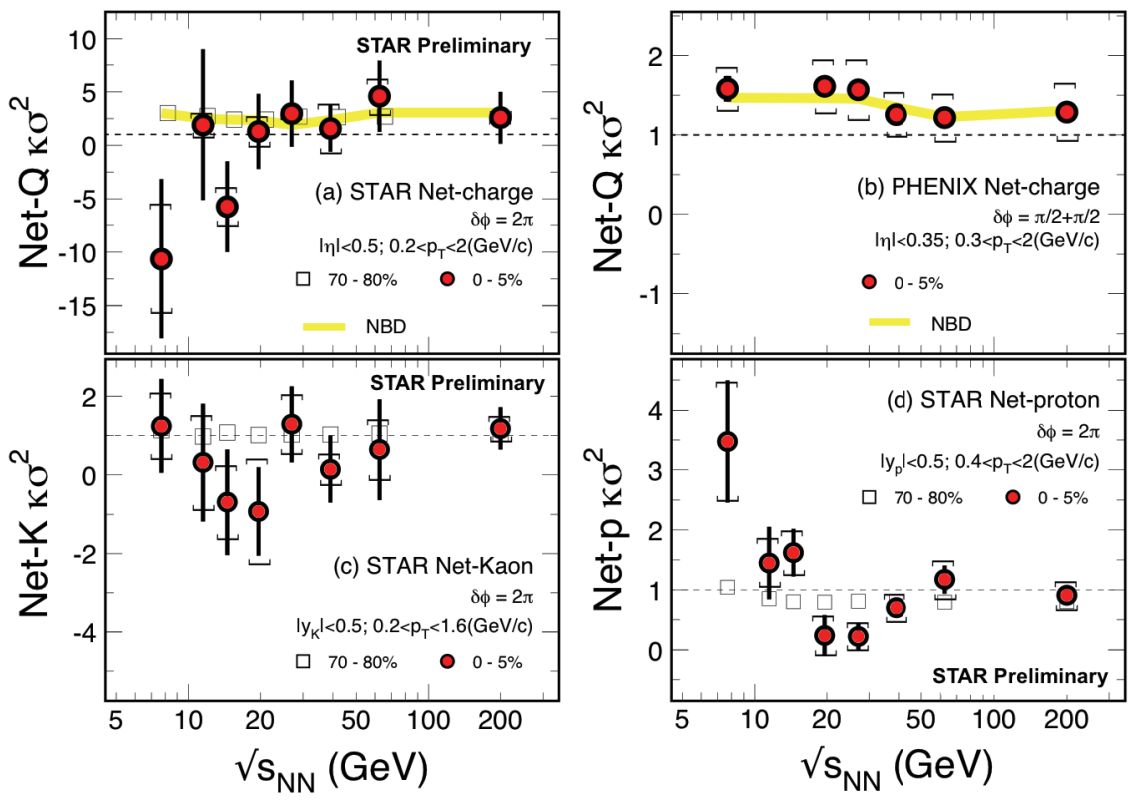

Figure 1. (Color online) The STAR measured energy dependence of $\kappa \sigma^{2}$ of net-proton, net-charge (top left) and net-kaon distributions in $\mathrm{Au}+\mathrm{Au}$ collisions at $\sqrt{s_{\mathrm{NN}}}=7.7,11.5,14.5,19.6,27,39,62.4$ and $200 \mathrm{GeV}$. The netcharge fluctuations measured by the PHENIX experiment in Au+Au collisions at $\sqrt{s_{\mathrm{NN}}}=7.7,19.6,27,39,62.4$ and $200 \mathrm{GeV}$ are shown in top right panel.The statistical and systematical error are shown in bars and brackets, respectively.

model calculations [22, 23]. At energies above $39 \mathrm{GeV}$, the results are close to Skellam expectation. For the STAR net-charge and net-kaon results, we did not observe non-monotonic behavior for $\kappa \sigma^{2}$ within current statistics. The expectations from negative binomial distribution can better describe the net-charge and net-kaon data than the Poisson (Skellam) expectations. The PHENIX net-charge results show smaller errors than the one measured by the STAR experiment. This is because the PHENIX detector has much smaller acceptance than the STAR detector and thus the width of the netcharge distributions measured by the PHENIX experiment is much narrower. On the other hand, the resonance decay effects are not excluded in the current experimental measurements of fluctuations of net-proton, net-kaon and net-charge multiplicity. However, based on the hadron resonance gas model calculation [24], the decay effects for net-proton $\kappa \sigma^{2}$ is small and at $2 \%$ level. But for the net-charge, the decay effects are large. The statistical error of cumulants $\left(\Delta\left(C_{n}\right)\right)$ are related to the width of the distribution as $\Delta\left(C_{n}\right) \sim \mathrm{O}\left(\sigma^{n}\right)[11,12]$. Thus, the wider is the distribution, the larger are statistical errors for the same number of events. It is predicted that the coupling strength of pions and kaons to the critical fluctuation is much smaller than protons. Thus, the fluctuation of net-protons will be more sensitive to the critical fluctuations than the net-charge and net-kaon [6].

\section{Detector upgrades and beam energy scan phase II at RHIC}

A second phase of the beam energy scan (BES-II) at RHIC has been planed in the years 2019-2020 and focusing on energies below $\sqrt{s_{\mathrm{NN}}}=20 \mathrm{GeV}$ [25]. This is to collect more number of events to confirm 
the trends observed in the BES-I. It will allow us to draw a solid conclusion and have more complete physics picture from various experimental measurements. The long beam bunches and stochastic
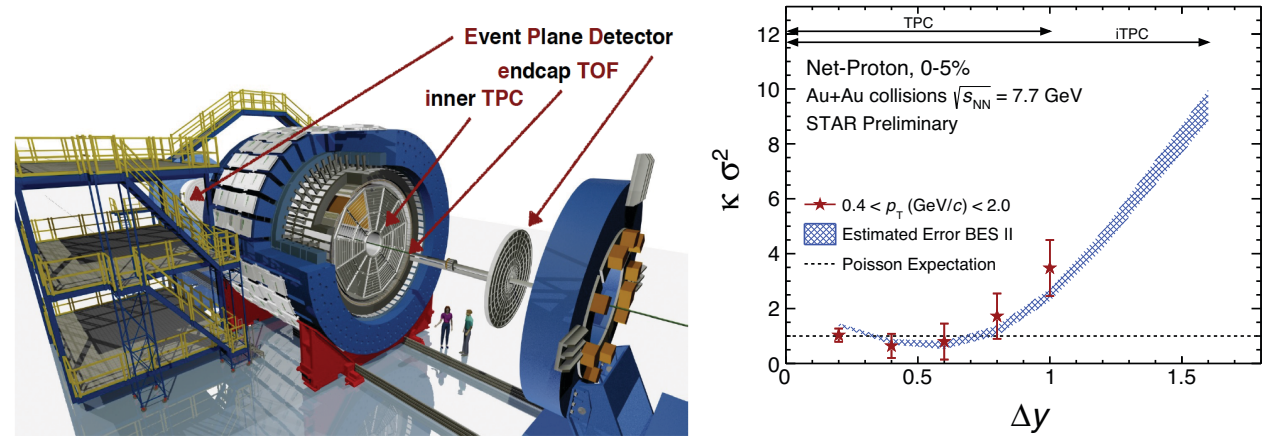

Figure 2. (Color online) Left: Upgrades of the STAR detector for the second phase of beam energy scan at RHIC. Right: Rapidity coverage dependence of the $\kappa \sigma^{2}$ of net-proton distribution in 0-5\% central Au+Au collisions at $\sqrt{s_{\mathrm{NN}}}=7.7 \mathrm{GeV}$. The blue band shows the expecting trend and statistical error for net-proton $\kappa \sigma^{2}$ at BES-II. For this analysis, the rapidity coverage can be extend to $|y|<0.8$ with iTPC upgrades.

electron cooling technique will be used to accelerate gold beams. It will increase the luminosity by a factor of 5-15 compared to the BES-I. Since the luminosity will decrease as decreasing the colliding energy, the increasing of the luminosity is much more necessary and important at low energies, such as $7.7 \mathrm{GeV}$. To study the QCD phase structure at high baryon density, operating the STAR detector at fixed target mode has been also proposed. In the BES-II, fixed target mode $\mathrm{Au}+\mathrm{Au}$ collisions allow us to have energy coverage from $\sqrt{s_{\mathrm{NN}}}=3 \mathrm{GeV}\left(\mu_{B}=720 \mathrm{MeV}\right)$ up to $7.7 \mathrm{GeV}$. In Fig.2 left, the inner TPC (iTPC) of STAR is to be upgraded to improve the energy loss resolution and can extend the pseudo-rapidity coverage from $|\eta|<1$ to $|\eta|<1.5$ [26]. It is also planed to install a end cap Time-ofFlight (eTOF) detector at the west end of the STAR TPC to extend the PID capability in the forward region [27]. The iTPC upgrade is very important to search for the criticality and study the dynamical evolution of the fluctuations by looking at the rapidity acceptance dependence for the fluctuations of the conserved quantities [28]. In the forward and backward region of STAR detector, a new Event Plane Detector (EPD) will be also built and used to replace the Beam-Beam Counters (BBC) detector for centrality and event plane determination, which can suppress the auto-correlations. In Fig.2 right, the blue band is the extrapolating from current measurements by assuming a power law behavior induced by critical fluctuations $\left(\kappa \sigma^{2} \propto N^{3}[29]\right)$.

\section{Summary}

The STAR and PHENIX experiments at RHIC have measured the fluctuation of net-proton, net-kaon, and net-charge multiplicity in Au+Au collisions at $\sqrt{s_{\mathrm{NN}}}=7.7,11.5,14.5,19.6,27,39,62.4$ and 200 $\mathrm{GeV}$. Different measurements/experiments are affected by acceptance, kinematic cuts, resonance decays, and other dynamical effects differently. To search for the signature of the QCD critical point, one needs to do careful studies. Non-monotonic energy dependence of $\kappa \sigma^{2}$ of the net-proton distributions is observed in the $0 \sim 5 \%$ most central $\mathrm{Au}+\mathrm{Au}$ collisions measured by the STAR experiment. This non-monotonic behavior cannot be described by various transport model calculations. The net-charge and net-kaon results are consistent with the binomial and Poisson expectations with current statistics. Experimentally, the second phase of the beam energy scan at RHIC has been planed in 2019-2020 
with upgraded detectors and increased luminosity to explore the phase structure focusing on energies below $20 \mathrm{GeV}$ with high precision.

\section{Acknowledgement}

The work was supported in part by the MoST of China 973-Project No.2015CB856901, NSFC under grant No. 11575069, 11221504.

\section{References}

[1] K. Rajagopal, F. Wilczek, ”At the Frontier of Particle Physics / Handbook of QCD”, Vol. 3 (World Scientific, 2001)

[2] Z. Fodor and S. Katz, JHEP 0203, 014 (2002); 0404, 050 (2004); R. V. Gavai, S. Gupta, Phys. Rev. D 71, 114014 (2005); 78, 114503 (2008).

[3] R. V. Gavai, arXiv:1404.6615.

[4] M. M. Aggarwal et al. (STAR Collaboration), arXiv: 1007.2613

[5] M. A. Stephanov, Phys. Rev. Lett. 102, 032301 (2009).

[6] C. Athanasiou et al., Phys. Rev. D 82, 074008 (2010).

[7] M. A. Stephanov, Phys. Rev. Lett. 107, 052301 (2011); B. Schaefer, M. Wagner, Phys. Rev. D 85, 034027 (2012).

[8] S. Gupta, X. Luo, B. Mohanty, H. G. Ritter, N. Xu, Science 332, 1525 (2011).

[9] R. V. Gavai and S. Gupta, Phys. Lett. B 696, 459 (2011).

[10] Y. Hatta and M. A. Stephanov, Phys. Rev. Lett. 91, 102003 (2003).

[11] X. Luo, J. Phys. G: Nucl. Part. Phys. 39, 025008 (2012).

[12] X. Luo, Phys. Rev. C 91, 034907 (2015).

[13] X. Luo, J. Xu, B. Mohanty, N. Xu, J. Phys. G 40, 105104 (2013).

[14] X. Luo (for the STAR Collaboration), J. Phys.: Conf. Ser. 316, 012003 (2011).

[15] Adam Bzdak and Volker Koch, Phys. Rev. C 86, 044904 (2012).

[16] Adam Bzdak and Volker Koch, Phys. Rev. C 91, 027901 (2015).

[17] L. Adamczyk et al., (STAR Collaboration), Phys. Rev. Lett. 112, 032302 (2014); Phys. Rev. Lett. 113, 092301 (2014);

[18] L. Adamczyk et al., (STAR Collaboration), Phys. Rev. Lett. 113, 092301 (2014).

[19] X. Luo (for the STAR Collaboration), PoS(CPOD2014)019, 2015. [arXiv: 1503.02558]

[20] X. Luo, Nucl. Phys. A 956, 75 (2016). [arXiv: 1512.09215].

[21] J. Thaeder, (for the STAR Collaboration), Nucl. Phys. A 956, 320 (2016). [arXiv:1601.00951]

[22] J. Xu, S. L. Yu, F. Lui, X. Luo, Phys. Rev. C 94, 024901(2016).

[23] S. He, X. Luo, Y. Nara, S. Esumi, N. Xu, Phys. Lett. B 762, 296 (2016).

[24] P. Garg et al., Phys. Lett. B 726, 691 (2013).

[25] STAR Note 0598: BES-II whitepaper: http://drupal.star.bnl.gov/STAR/starnotes/public/sn0598

[26] STAR Note 0619: iTPC proposal: http://drupal.star.bnl.gov/STAR/starnotes/public/sn0619

[27] L. Adamczyk et al., STAR Collaboration, arXiv:1609.05102

[28] Masakiyo Kitazawa, Nucl. Phys. A 942, 65 (2015).

[29] Bo Ling and Mikhail A. Stephanov, Phys. Rev. C 93, 034915 (2016). [arXiv: 1512.09125]. 